\title{
Molecular Clips Form Isostructural Dimeric Aggregates From Benzene to Water - Supporting Information
}

\author{
by Anxin Wu, Pritam Mukhopadhyay, Arindam Chakraborty, \\ James C. Fettinger, and Lyle Isaacs*
}

Table of Contents

Pages

Table of Contents

S1

Details of the X-ray Structure Determinations of 2

$\mathrm{S} 2-\mathrm{S} 4$

Figure S1. MMFF minimized Structure of $\mathbf{8 \bullet 8}$

S5

Table S1. Solvent dependent ${ }^{1}$ H NMR for $\mathbf{2} \cdot \mathbf{2}$

S6

Models Used in Scientist

S6 - S7

${ }^{1} \mathrm{H}$ and ${ }^{13} \mathrm{C}$ NMR spectra for $\mathbf{3}-\mathbf{7}, \mathbf{1 2}$, and $\mathbf{1 3}$

S8 - S21 


\section{Details of the X-ray crystallographic structure determination of 2.}

A colorless block with approximate orthogonal dimensions $0.29 \times 0.18 \times 0.12 \mathrm{~mm}^{3}$ was placed and optically centered on the Bruker SMART CCD system at $-100^{\circ} \mathrm{C}$. The initial unit cell was indexed using a least-squares analysis of a random set of reflections collected from three series of $0.3^{\circ}$ wide $\omega$-scans, 10 seconds per frame, and 25 frames per series that were well distributed in reciprocal space. Data frames were collected $[\mathrm{MoK} \alpha]$ with $0.3^{\circ}$ wide $\omega$-scans, 30 seconds per frame and 606 frames per series. Five data series were collected at varying $\varphi$ angles $\left(\varphi=0^{\circ}, 72^{\circ}\right.$, $\left.144^{\circ}, 216^{\circ}, 288^{\circ}\right)$, including a partial repeat of the first series, 200 frames, for decay purposes. The crystal to detector distance was $4.456 \mathrm{~cm}$, thus providing a complete sphere of data to $2 \theta_{\max }=55.0^{\circ}$. A total of 444821 reflections were collected and corrected for Lorentz and polarization effects and absorption using Blessing's method as incorporated into the program SADABS ${ }^{1,} \quad 2$ with 11486 unique $[\mathrm{R}$ (int) $=0.0525]$

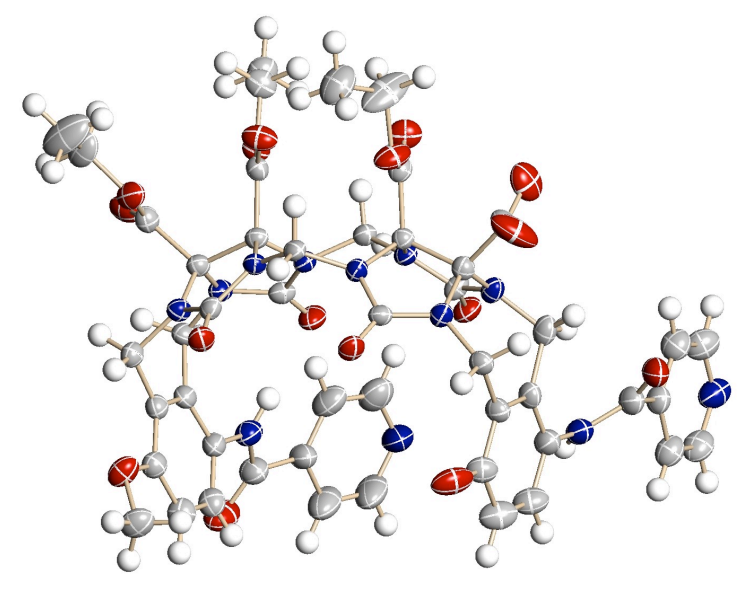

\section{Structural determination and Refinement:}

All crystallographic calculations were performed on a Personal computer (PC) with a Pentium $1.80 \mathrm{GHz}$ processor and $512 \mathrm{MB}$ of extended memory. The SHELXTL ${ }^{3}$ program package was implemented to determine the probable space group and set up the initial files. System symmetry, systematic absences and intensity statistics indicated the centrosymmetric monoclinic non-standard space group $\mathrm{P} 2 / \mathrm{c}$ (no. 14). The structure was determined by direct methods with the successful location of nearly the entire molecule using the program $\mathrm{XS}^{4}$. The structure was refined with $\mathrm{XL}^{5}$. Least-squares difference-Fourier cycling was required to locate the remaining, full occupancy and disordered, non-hydrogen atoms. Several disordered regions of the molecule were optimized. All full occupancy non-hydrogen atoms were refined anisotropically. The hydrogen atoms were calculated and placed in idealized positions, then allowed to refine freely during the final refinement cycles. A solvent region near one of the terminal groups was optimized and found to be composed of a partial occupancy methanol molecule and also multiple water molecules; the water molecules did not have their hydrogen atoms located. The final structure was refined to convergence $[\Delta / \sigma \leq 0.001]$ with $\mathrm{R}(\mathrm{F})=9.48 \%, \omega \mathrm{W}\left(\mathrm{F}^{2}\right)=15.95 \%$, $\mathrm{GOF}=1.096$ for all 11486 unique reflections $\left[\mathrm{R}(\mathrm{F})=5.21 \%\right.$, $\mathrm{wR}\left(\mathrm{F}^{2}\right)=14.26 \%$ for those 7274 data with Fo $>4 \sigma(\mathrm{Fo})]$. The final difference-Fourier map was featureless indicating that the structure is both correct and complete.

The function minimized during the full-matrix least-squares refinement was $\Sigma \mathrm{w}\left(\mathrm{Fo}^{2}-\mathrm{Fc}^{2}\right)$ where $\mathrm{w}=1 /\left[\sigma^{2}\left(\mathrm{Fo}^{2}\right)+(0.0865 * \mathrm{P})^{2}+0.2511 * \mathrm{P}\right]$ and $\mathrm{P}=\left(\max \left(\mathrm{Fo}^{2}, 0\right)+2 * \mathrm{Fc}^{2}\right) / 3$. An empirical correction for extinction was also attempted but found to be negative and therefore not applied. 


\section{References:}

1) An Empirical Correction for Absorption Anisotropy, Blessing, R. H. (1995). Acta Cryst., A51, 33-38.

2) Sheldrick, G.M., SADABS 'Siemens Area Detector Absorption Correction' Universität Göttingen: Göttingen, Germany, 1996.

3) Sheldrick, G.M., (1994). SHELXTL/PC. Version 5.03. Siemens Analytical X-ray Instruments Inc., Madison, Wisconsin, USA.

4) Phase Annealing in SHELX-90: Direct Methods for Larger Structures, Sheldrick, G. M., (1990). Acta Cryst. A46, 467-473.

5) Sheldrick, G.M., (1993). Shelx193 Program for the Refinement of Crystal Structures. University of Göttingen, Germany. 
Table 1. Crystal data and structure refinement for $\left[\mathrm{C}_{52} \mathrm{H}_{52} \mathrm{~N}_{12} \mathrm{O}_{16}\right]\left[\mathrm{CH}_{3} \mathrm{OH}\right]_{0.15}\left[\mathrm{H}_{2} \mathrm{O}\right]_{0.85}$.

Identification code

Empirical formula

Formula weight

Temperature

Wavelength

Crystal system

Space group

Unit cell dimensions

Volume

$\mathrm{Z}$

Density (calculated)

Absorption coefficient

$\mathrm{F}(000)$

Crystal size

Theta range for data collection

Index ranges

Reflections collected

Independent reflections

Completeness to theta $=27.50^{\circ}$

Absorption correction

Max. and min. transmission

Refinement method

Data / restraints / parameters

Goodness-of-fit on $\mathrm{F}^{2}$

Final $\mathrm{R}$ indices [I>2sigma(I)]

$\mathrm{R}$ indices (all data)

Largest diff. peak and hole
1040 cffmi

C52.15 H54.30 N12 O17

1121.18

173(2) K

$0.71073 \AA$

Monoclinic

$\mathrm{P} 2(1) / \mathrm{c}$

$$
\begin{aligned}
& a=20.2117(7) \AA \quad \alpha=90^{\circ} \text {. } \\
& \mathrm{b}=15.3609(5) \AA \\
& \beta=111.5020(10)^{\circ} \text {. } \\
& \mathrm{c}=17.3442(6) \AA \\
& \gamma=90^{\circ} \text {. }
\end{aligned}
$$

$5010.1(3) \AA^{3}$

4

$1.486 \mathrm{Mg} / \mathrm{m}^{3}$

$0.113 \mathrm{~mm}^{-1}$

2349

$0.29 \times 0.18 \times 0.12 \mathrm{~mm}^{3}$

1.71 to $27.50^{\circ}$.

$-26<=\mathrm{h}<=26,-19<=\mathrm{k}<=19,-22<=\mathrm{l}<=22$

44821

$11486[\mathrm{R}(\mathrm{int})=0.0525]$

$99.9 \%$

Empirical, SADABS (multi-scan)

0.9867 and 0.9682

Full-matrix least-squares on $\mathrm{F}^{2}$

11486 / 8 / 753

1.096

$\mathrm{R} 1=0.0521, \mathrm{wR} 2=0.1426$ [7274 Data]

$\mathrm{R} 1=0.0948, \mathrm{wR} 2=0.1595$

0.366 and -0.343 e. $\AA^{-3}$ 

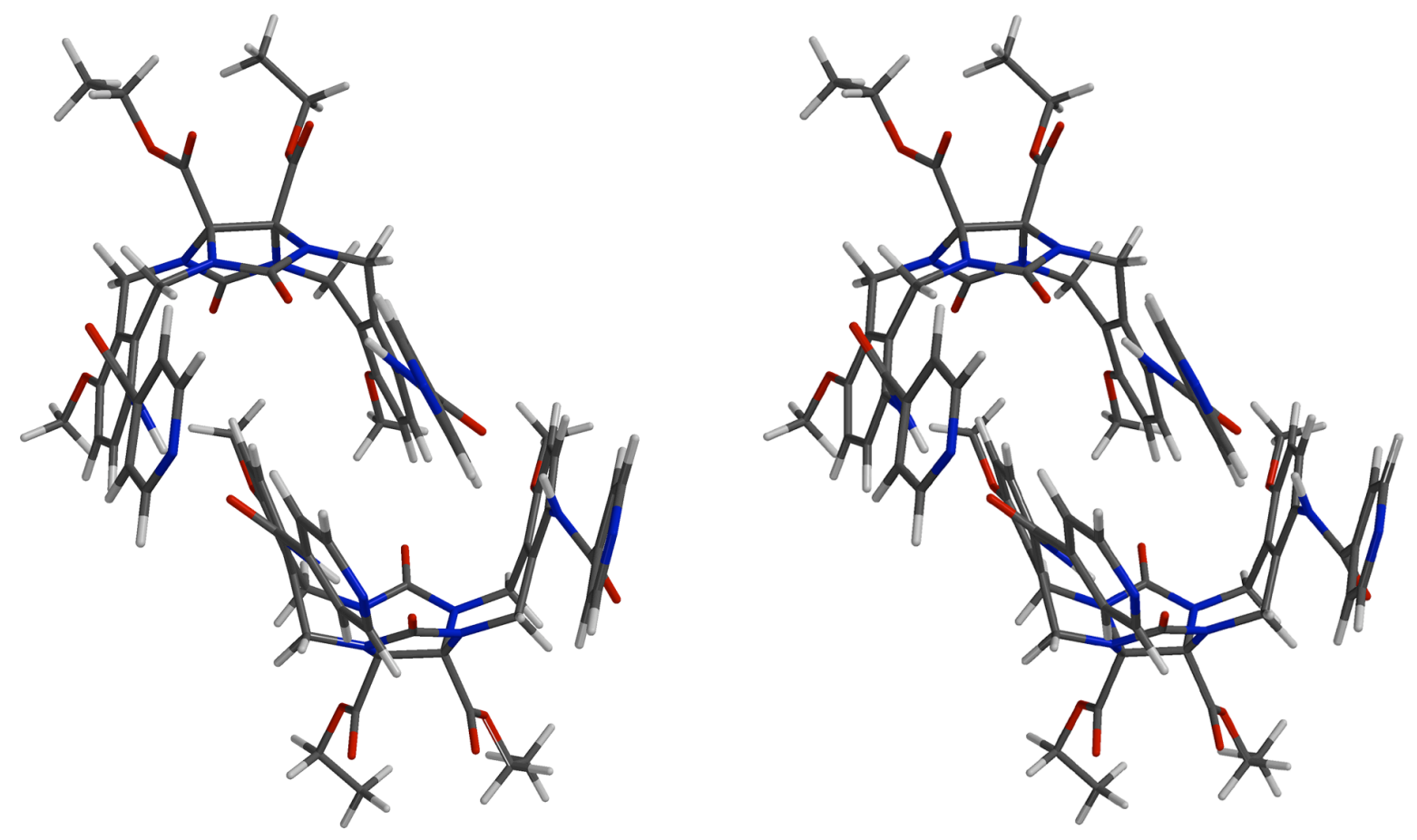

Figure S1. Stereoview of the MMFF minimized structure of $\mathbf{8} \bullet \mathbf{8}$. 
Table S1. Selected ${ }^{1} \mathrm{H}$ NMR data recorded for $\mathbf{2 \bullet 2}$ in different solvents. Conditions: $400 \mathrm{MHz}$, $\mathrm{CDCl}_{3}, 298 \mathrm{~K}, 10 \mathrm{mM}$.

\begin{tabular}{|c|c|c|c|c|c|c|}
\hline \multirow{3}{*}{$\begin{array}{l}\text { Solvent } \\
\mathrm{CDCl}_{3}\end{array}$} & \multicolumn{2}{|c|}{$\delta \mathrm{NH}$} & \multicolumn{2}{|c|}{$\delta \mathrm{Ar}_{\text {out }}{ }^{\mathrm{c})}$} & \multirow{2}{*}{$\begin{array}{c}\delta \mathrm{Ar}_{\mathrm{in}}{ }^{\mathrm{c}}{ }^{2} \\
\mathrm{H}_{\mathrm{b}}, \quad \mathrm{H}_{\mathrm{c}},\end{array}$} & \multirow[t]{2}{*}{$\left.K_{\mathrm{s}}\left(\mathrm{M}^{-1}\right)^{\mathrm{f}}\right)$} \\
\hline & & $\mathrm{H}_{\mathrm{a}}$ & & $\mathrm{H}_{\mathrm{c}}$ & & \\
\hline & 9.76 & 9.13 & 6.32 & 6.50 & $5.54 \quad 5.41$ & $>9 \times 10^{5}$ \\
\hline $\mathrm{C}_{6} \mathrm{D}_{5} \mathrm{CD}_{3}{ }^{\mathrm{a})}$ & 9.96 & 9.46 & 6.12 & 6.71 & $5.97 \quad 5.79$ & $>9 \times 10^{5}$ \\
\hline $\mathrm{C}_{6} \mathrm{D}_{6}{ }^{\mathrm{a})}$ & 10.06 & 9.55 & 6.1 & 6.78 & $6.06 \quad 5.89$ & $>9 \times 10^{5}$ \\
\hline $\mathrm{CD}_{2} \mathrm{Cl}_{2}{ }^{\mathrm{b})}$ & 9.79 & 9.19 & 6.38 & 6.53 & $5.54 \quad 5.43$ & 5700 \\
\hline $\mathrm{CD}_{3} \mathrm{CN}^{\mathrm{b})}$ & 9.65 & 9.05 & 6.5 & 6.62 & $5.60-5.40$ (br.) & 3350 \\
\hline $\mathrm{CD}_{3} \mathrm{COCD}_{3}{ }^{\mathrm{b})}$ & 9.77 & 9.21 & 6.5 & 6.72 & $5.60-5.40$ (br.) & 20300 \\
\hline $\mathrm{CD}_{3} \mathrm{OD}^{\mathrm{b})}$ & 10.03 & 9.20 & 6.60 & 6.75 & $\begin{array}{ll}5.54 & 5.43\end{array}$ & $>9 \times 10^{5}$ \\
\hline $\mathrm{CD}_{3} \mathrm{SOCD}_{3}{ }^{\mathrm{d})}$ & 10.43 & - & 7.02 & 7.19 & - & - \\
\hline $\mathrm{D}_{2} \mathrm{O}^{\mathrm{e})}$ & 9.80 & 9.37 & 6.6 & 6.74 & $5.60-5.40$ (br.) & 36300 \\
\hline
\end{tabular}

${ }^{\text {a) }}$ Recorded for a saturated solution.

b) Recorded at $-30{ }^{\circ} \mathrm{C}$.

c) Assignments are based on cross peaks observed in the COSY and ROESY spectra.

d) Compound 2 is monomeric in $\mathrm{CD}_{3} \mathrm{SOCD}_{3}$.

e) Measured using 2a in 15:85 $\mathrm{D}_{2} \mathrm{O}: \mathrm{H}_{2} \mathrm{O}(\mathrm{v}: \mathrm{v})$ at $5{ }^{\circ} \mathrm{C}$.

f) We estimate an error of $\pm 10 \%$ in the values of $K_{\mathrm{s}}$.

Models Used in Scientist. All calculations were performed on a Dell Precision 620 workstation with 512 MB of RAM and dual Pentium III processors running Scientist under Windows 2000 Professional.

Model used to calculate the self-association constant given in the text:

// MicroMath Scientist Model File

IndVars: Atot

DepVars: Deltaobsa, Deltaobsb, Deltaobsc, Deltaobsd, Deltaobse

Params: Deltazeroa, Deltasata, Deltasatb, Deltazerob, Deltasatc, Deltazeroc, Deltasatd,

Deltazerod, Deltasate, Deltazeroe, Keq

Keq $=$ Adimer $/$ Amono $^{\wedge} 2$

Atot $=$ Amono $+2 *$ Adimer

Deltaobsa $=$ Deltazeroa $+($ Deltasata-Deltazeroa $) *((2 *$ Adimer $) /$ Atot $)$ 
Deltaobsb=Deltazerob $+($ Deltasatb-Deltazerob $) *((2 *$ Adimer $) /$ Atot $)$

Deltaobsc $=$ Deltazeroc $+($ Deltasatc-Deltazeroc $) *((2 *$ Adimer $) /$ Atot $)$

Deltaobsd=Deltazerod $+($ Deltasatd-Deltazerod $) *((2 *$ Adimer $) /$ Atot $)$

Deltaobse $=$ Deltazeroe $+($ Deltasate-Deltazeroe $) *((2 *$ Adimer $) /$ Atot $)$

//Parameter Values

Deltazeroa $=5.7$

Deltazerob $=4.7$

Deltazeroc $=5.1$

Deltasata $=5.5$

Deltasatb $=4.9$

Deltasatc $=4.9$

Deltazerod $=4.9$

Deltasatd $=4.6$

Deltazeroe $=4.9$

Deltasate $=4.6$

Keq $=2000$

$0<$ Adimer $<0.5^{*}$ Atot

$0<$ Amono $<$ Atot

$* * *$

Model used for the simulation shown in Figure 7:

// MicroMath Scientist Model File

IndVars: Kab

DepVars: ChiAA, ChiAB, ChiBB, ChiAfree, ChiBfree

Params: Atot, Btot, Kaa, Kbb

$\mathrm{Kaa}=\mathrm{ConcAA} /($ ConcAfree $*$ ConcAfree $)$

$\mathrm{Kbb}=\mathrm{ConcBB} /(\mathrm{ConcB}$ free $*$ ConcBfree $)$

$\mathrm{Kab}=\mathrm{Conc} \mathrm{AB} /($ ConcAfree $*$ ConcBfree $)$

Atot $=2 *$ ConcAA + ConcAfree + Conc $A B$

$\mathrm{Btot}=2 * \mathrm{ConcBB}+$ ConcBfree + Conc $\mathrm{AB}$

ChiAA $=(2 *$ ConcAA $) /$ Atot

Chi $\mathrm{AB}=$ Conc $\mathrm{AB} /$ Atot

$\mathrm{ChiBB}=(2 *$ ConcBB $) /$ Btot

ChiAfree $=$ ConcAfree $/$ Atot

ChiBfree $=$ ConcBfree $/$ Btot

$0<$ ConcAfree $<$ Atot

$0<$ ConcBfree $<$ Btot

$0<$ ConcAA $<$ Atot

$0<$ ConcBB $<$ Btot

$0<$ Conc $\mathrm{AB}<$ Atot

$* * * *$ 


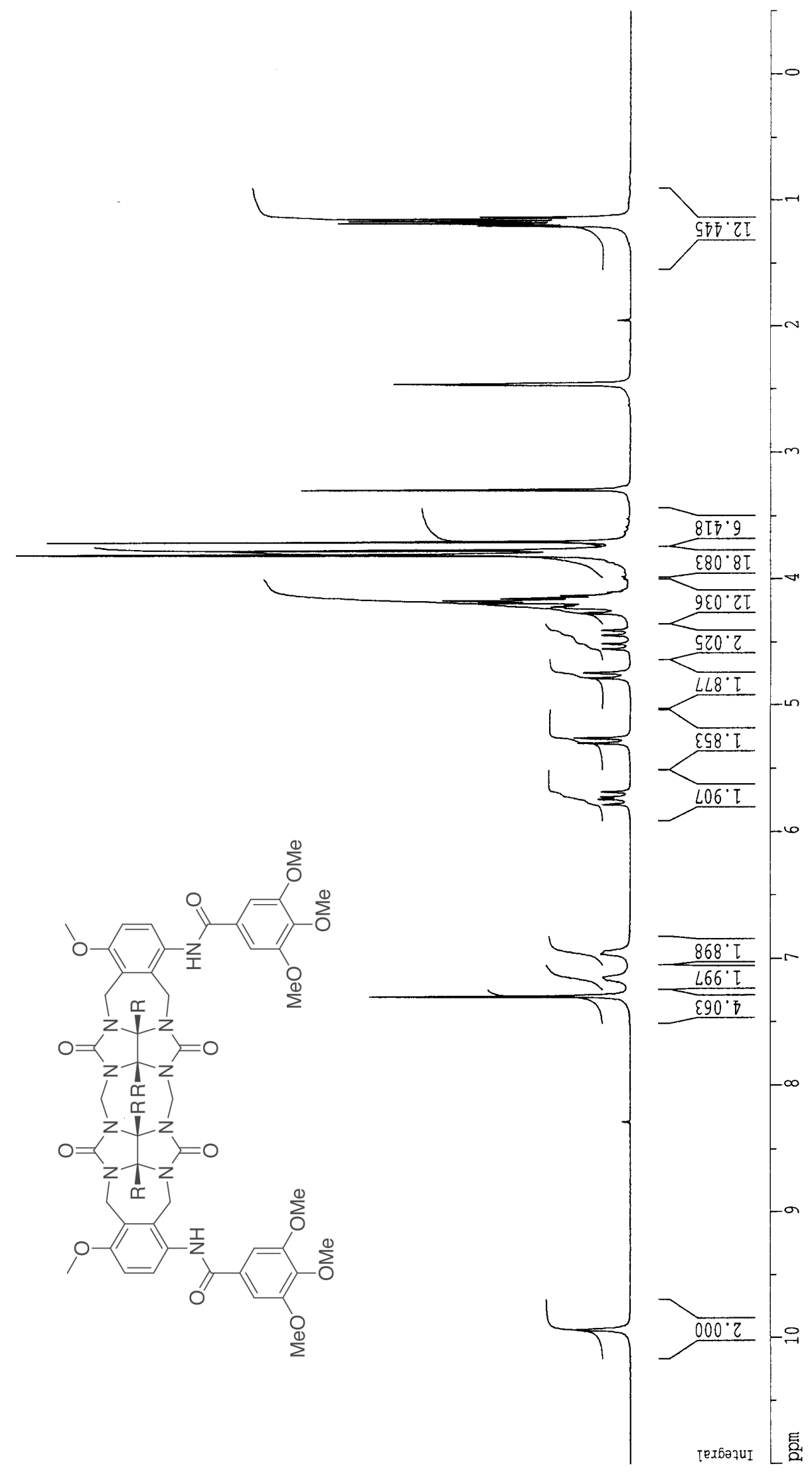




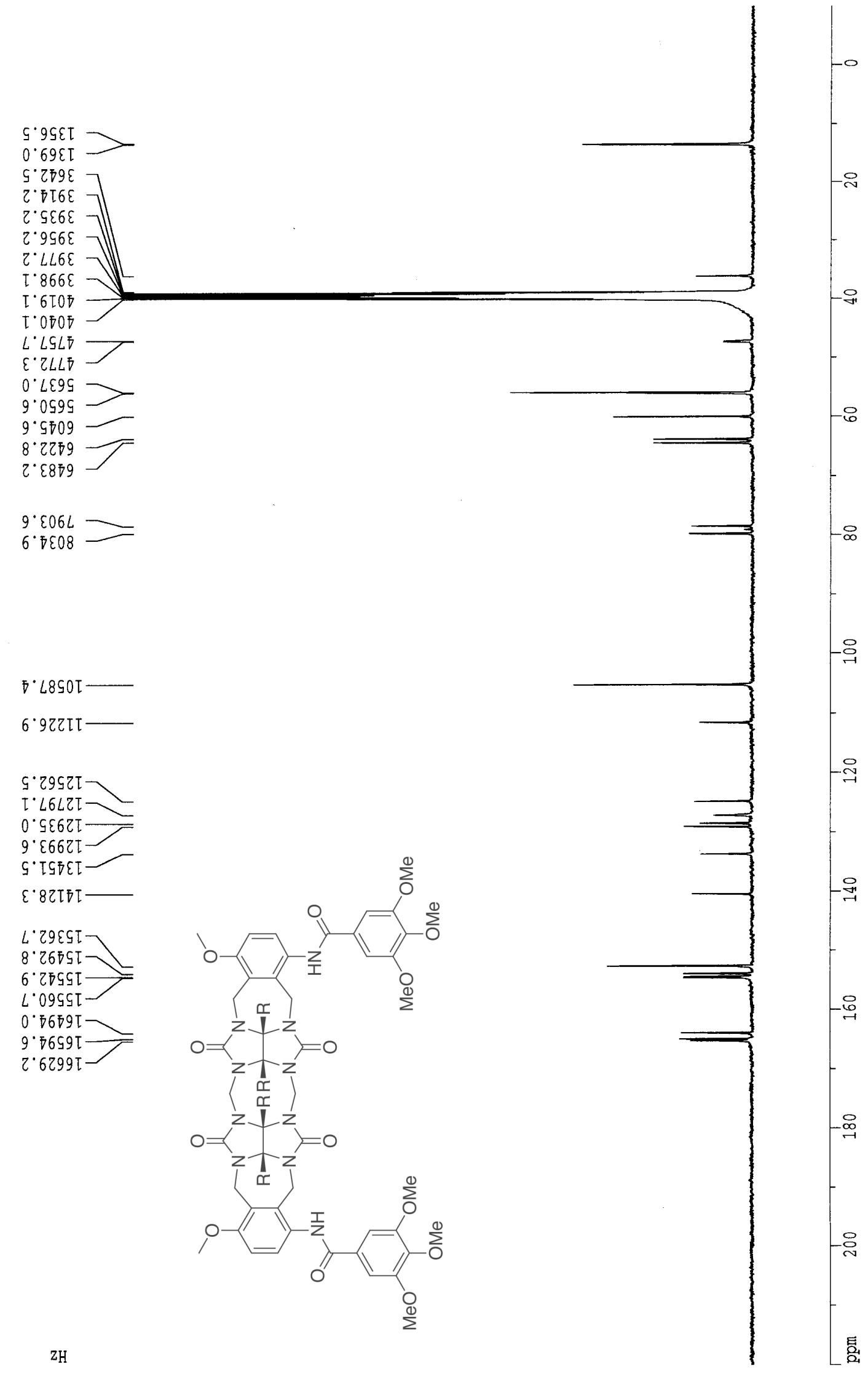



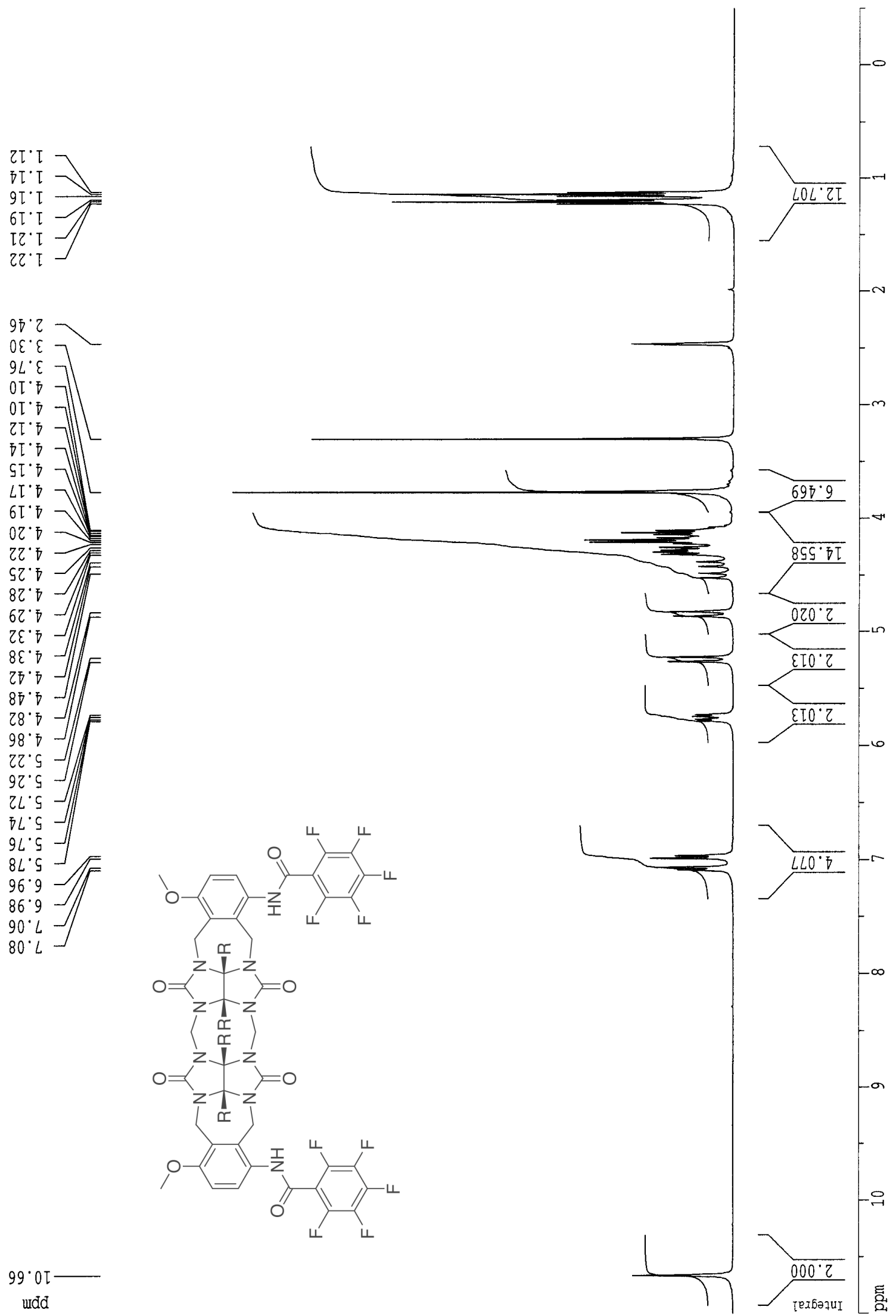

$99^{\circ} 0 \tau$

wdd 


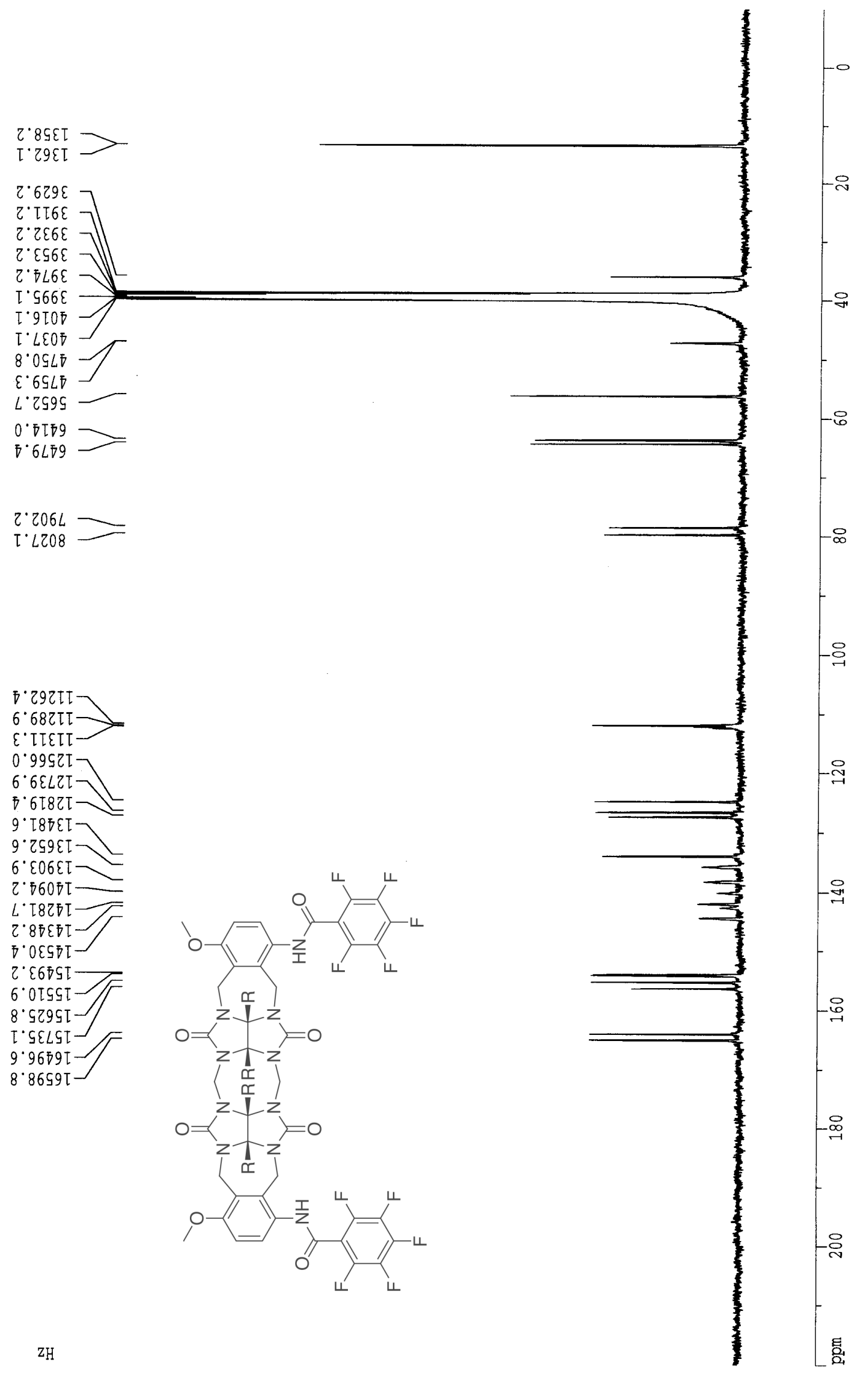




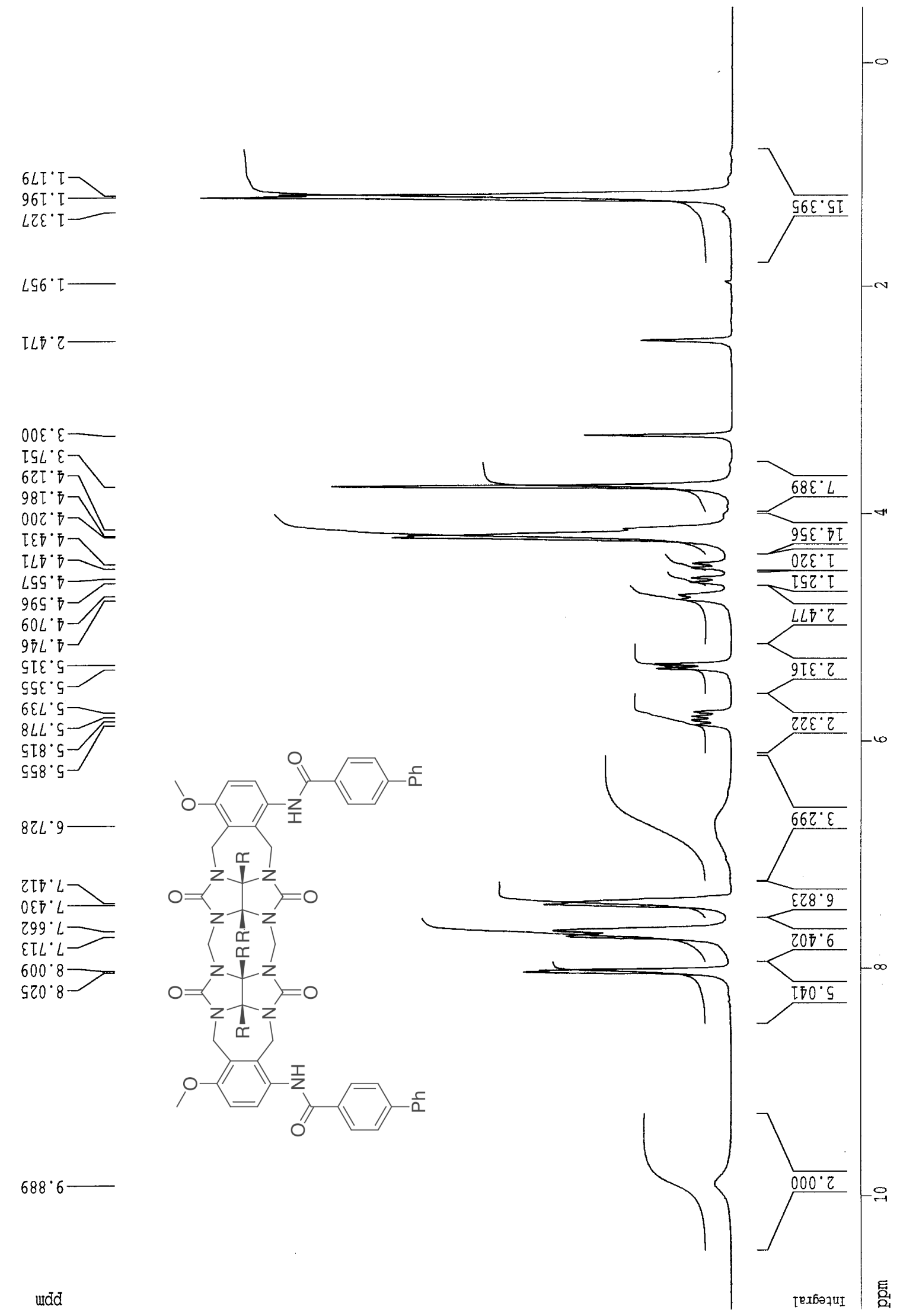


I.807 $\longrightarrow$

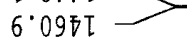

S. STLE

S. $000 \bar{D}$

S. 5200

5.9700

$\sigma^{\circ} \angle 90 D$

万. 880 T

5.60T万

D.OETE

¿'L78D

$L \cdot \varepsilon \angle 8 D$

$0^{\circ} 20 L 5$

$9^{\circ}$ ETS9

०. $2999=$

8.986L

ह' T2T8
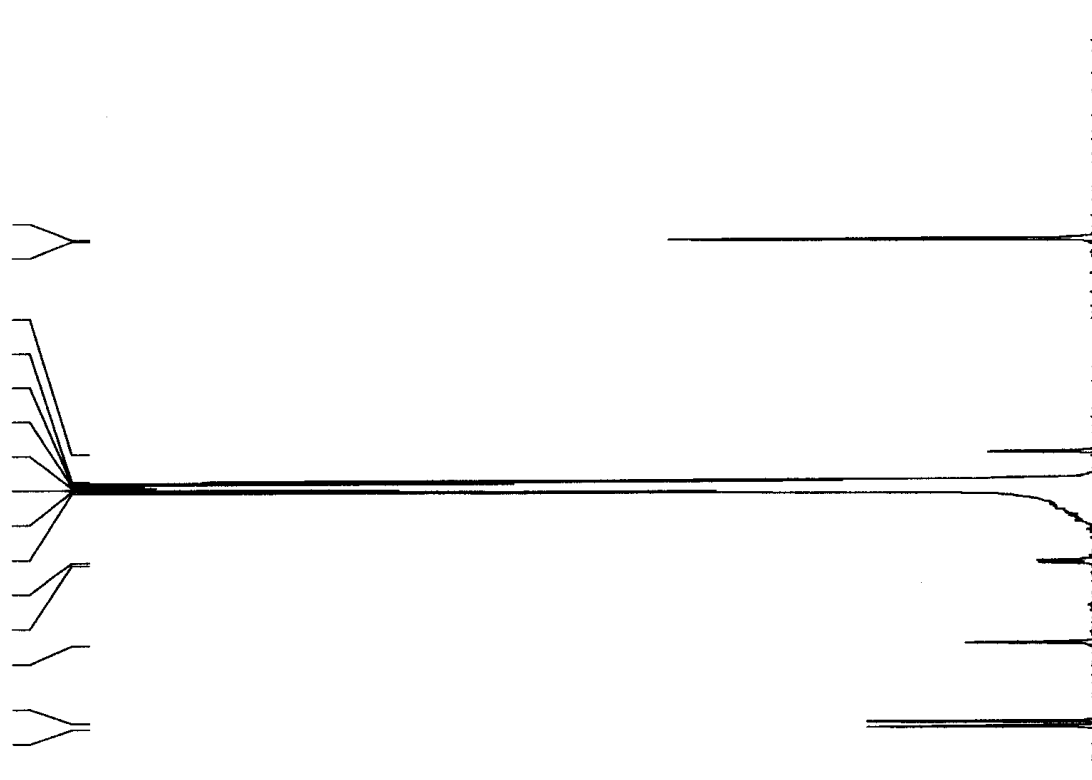

年

-o
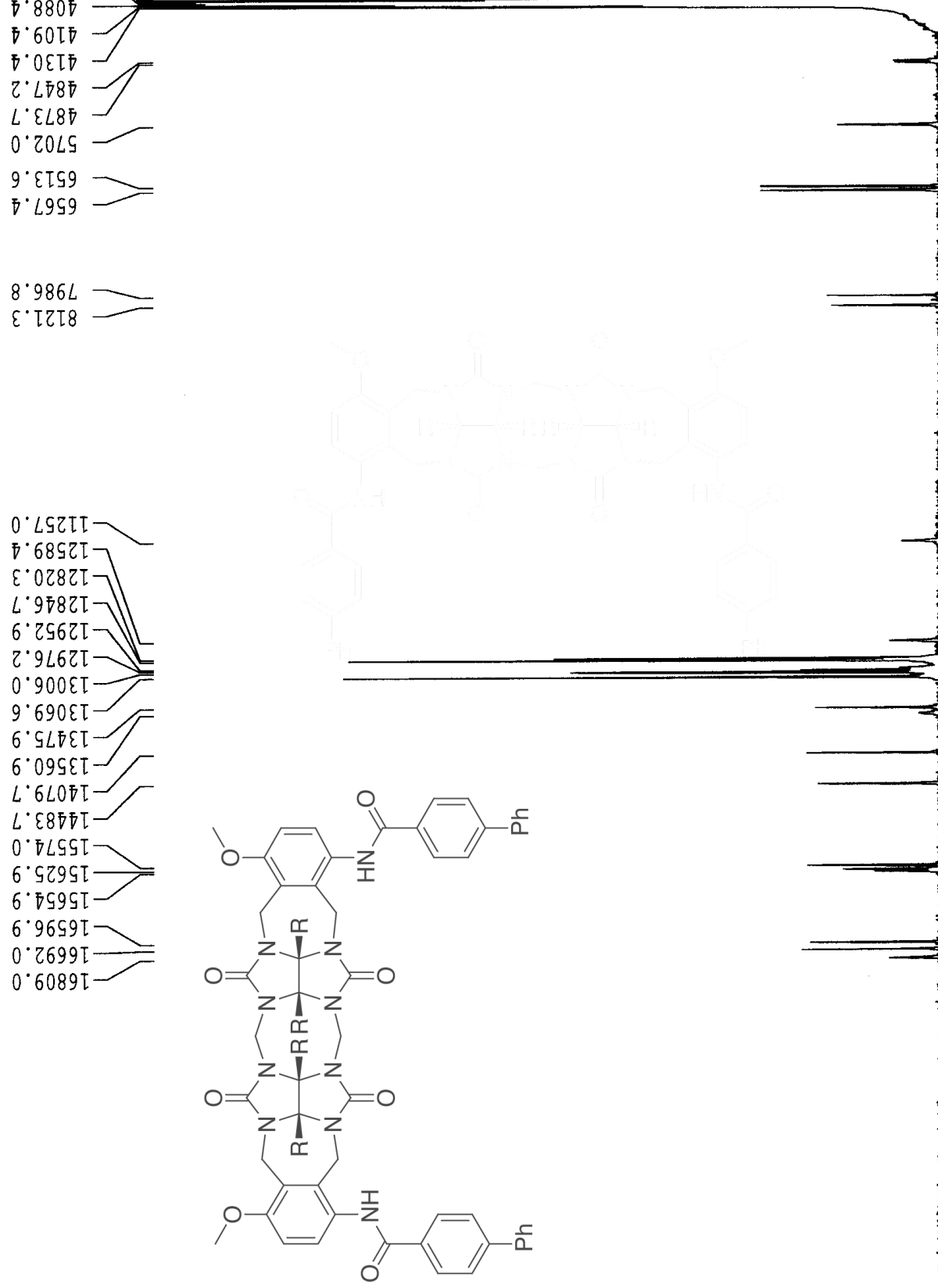

$-$

유 


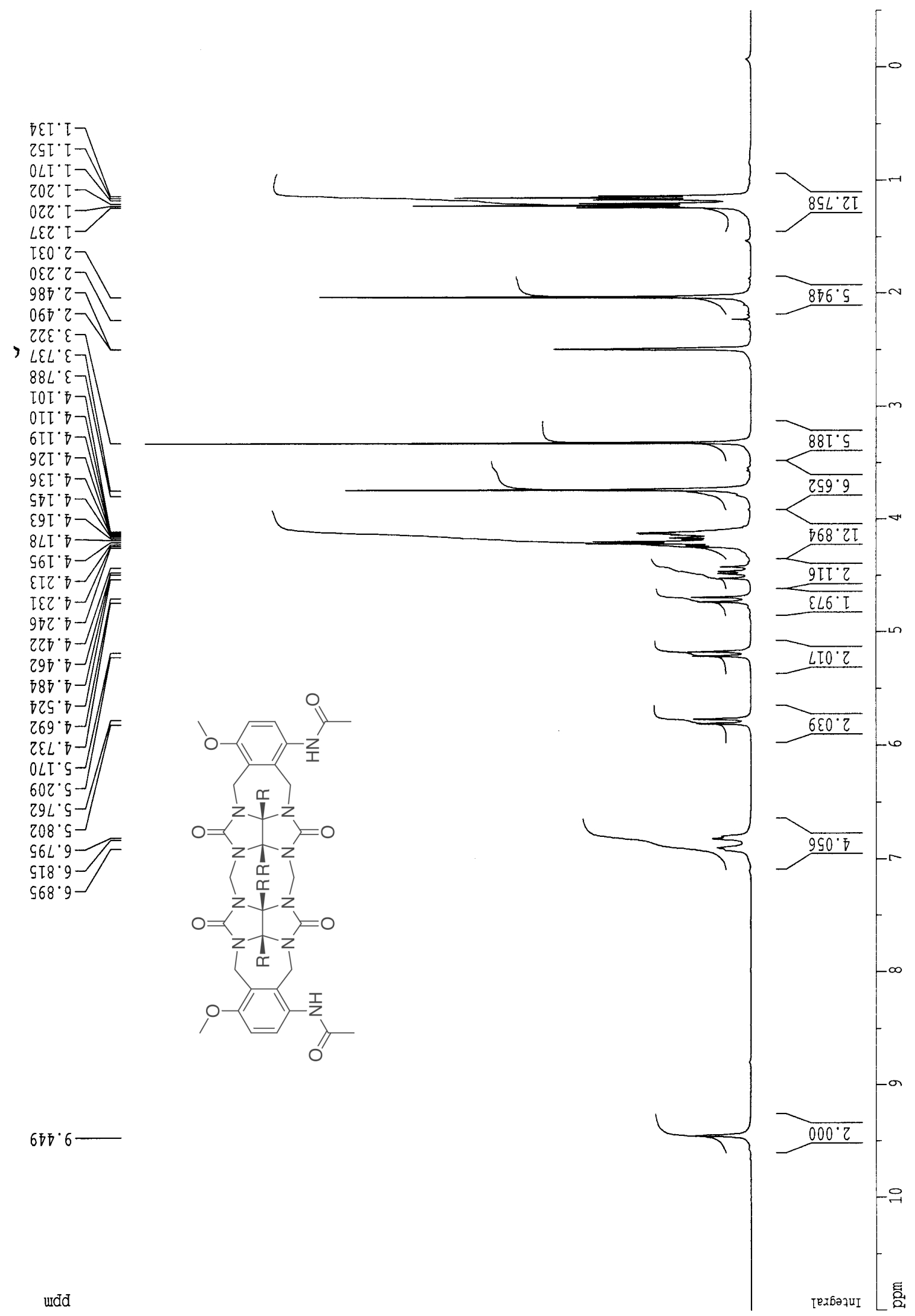



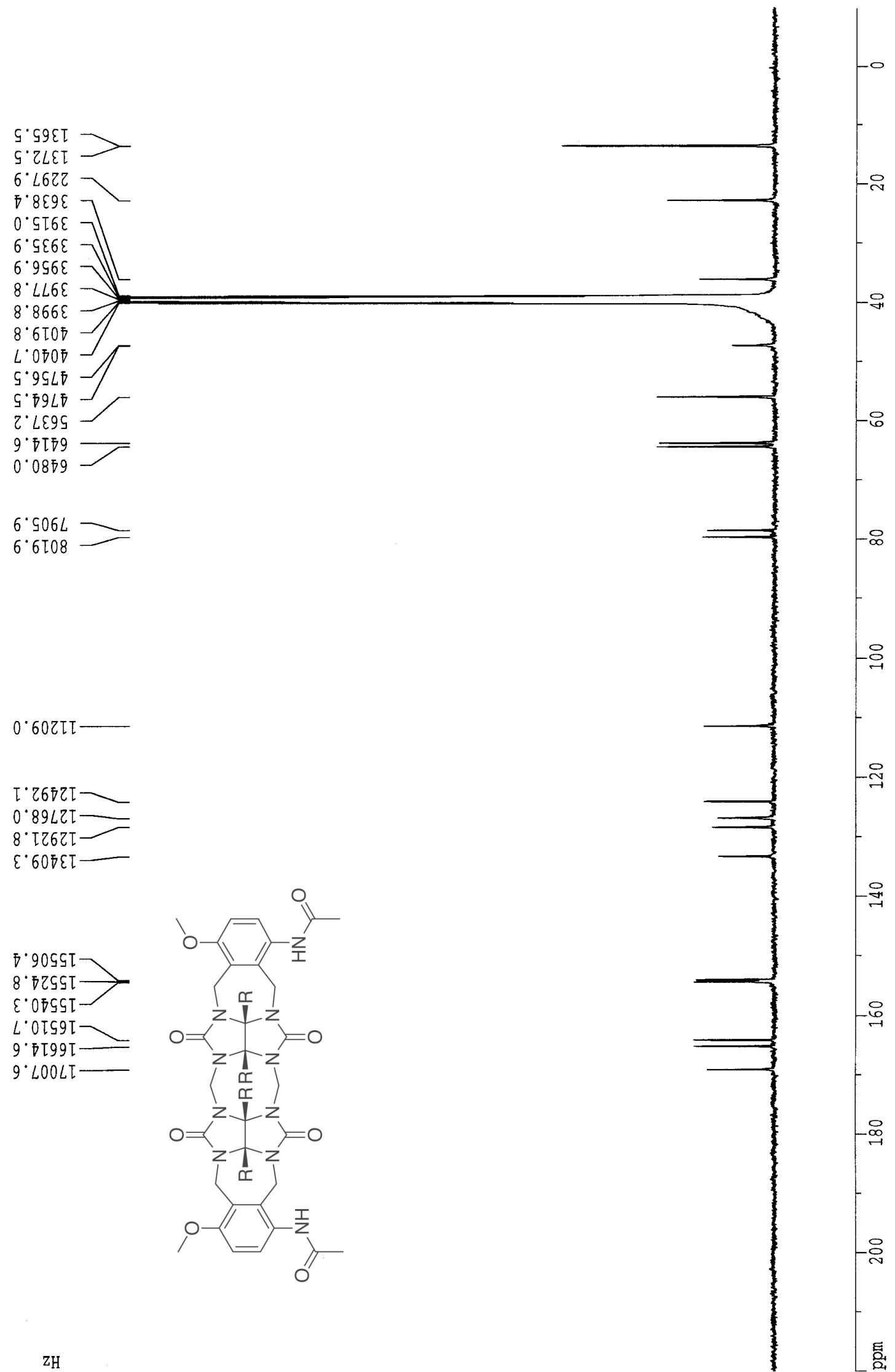


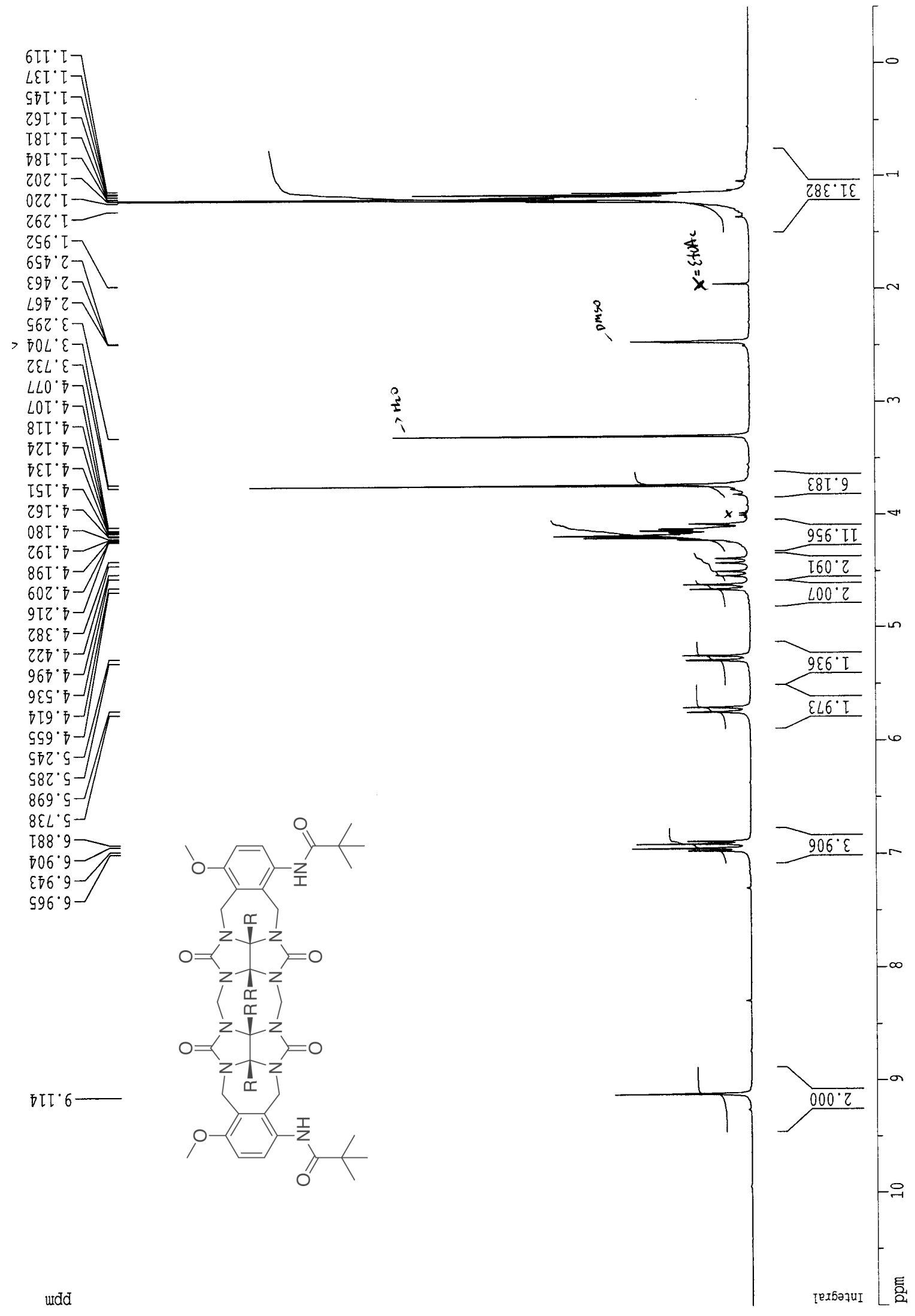




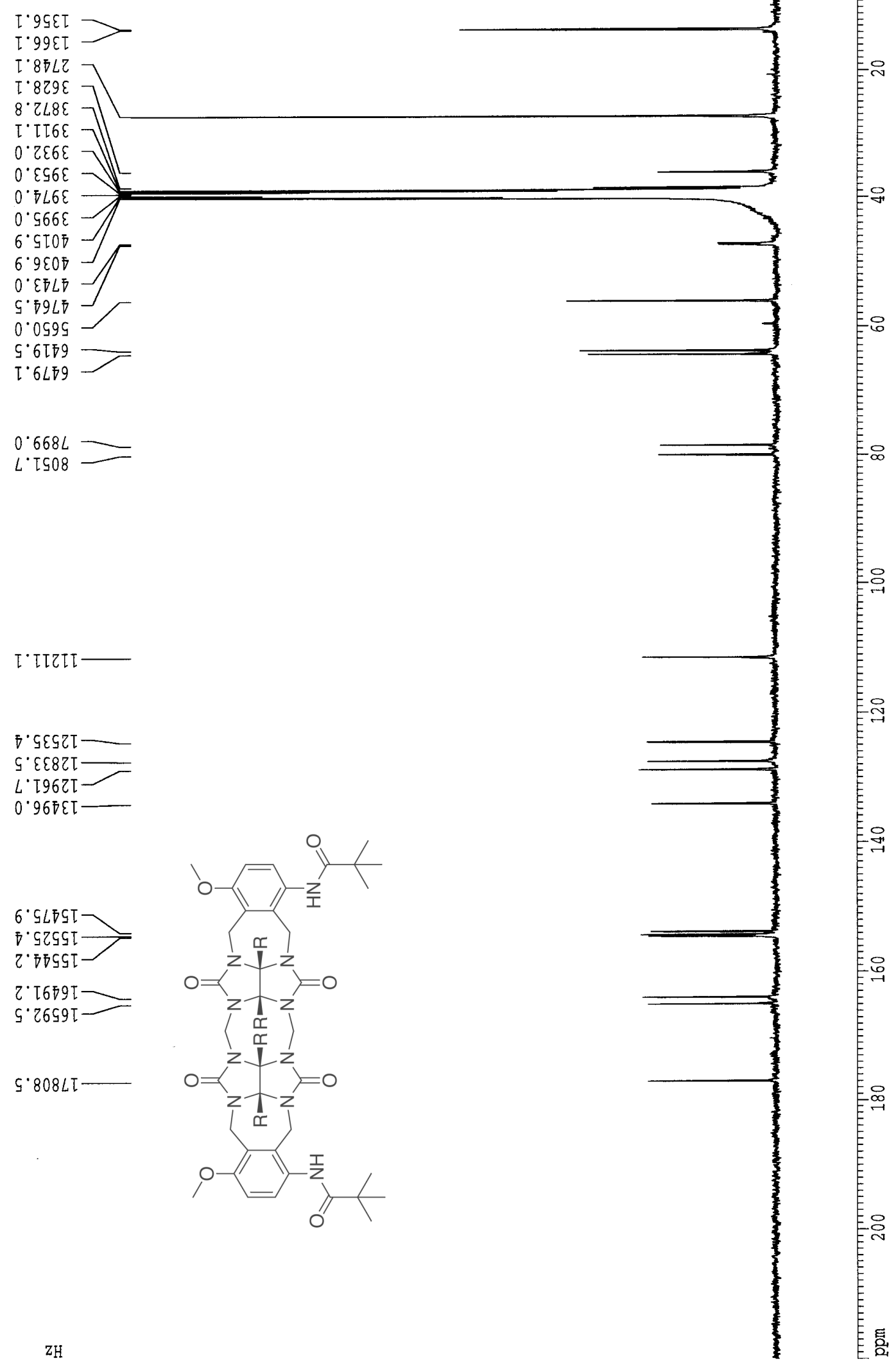




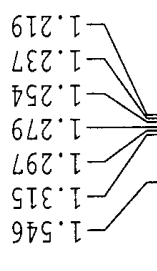

$720^{\circ} 2$

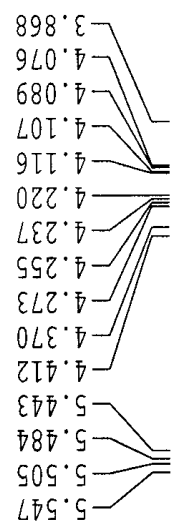

E9L' 9

$98 \mathrm{~L} \cdot 9$

$6 \varepsilon \tau^{\circ} L$

IOL $L L C$

udd

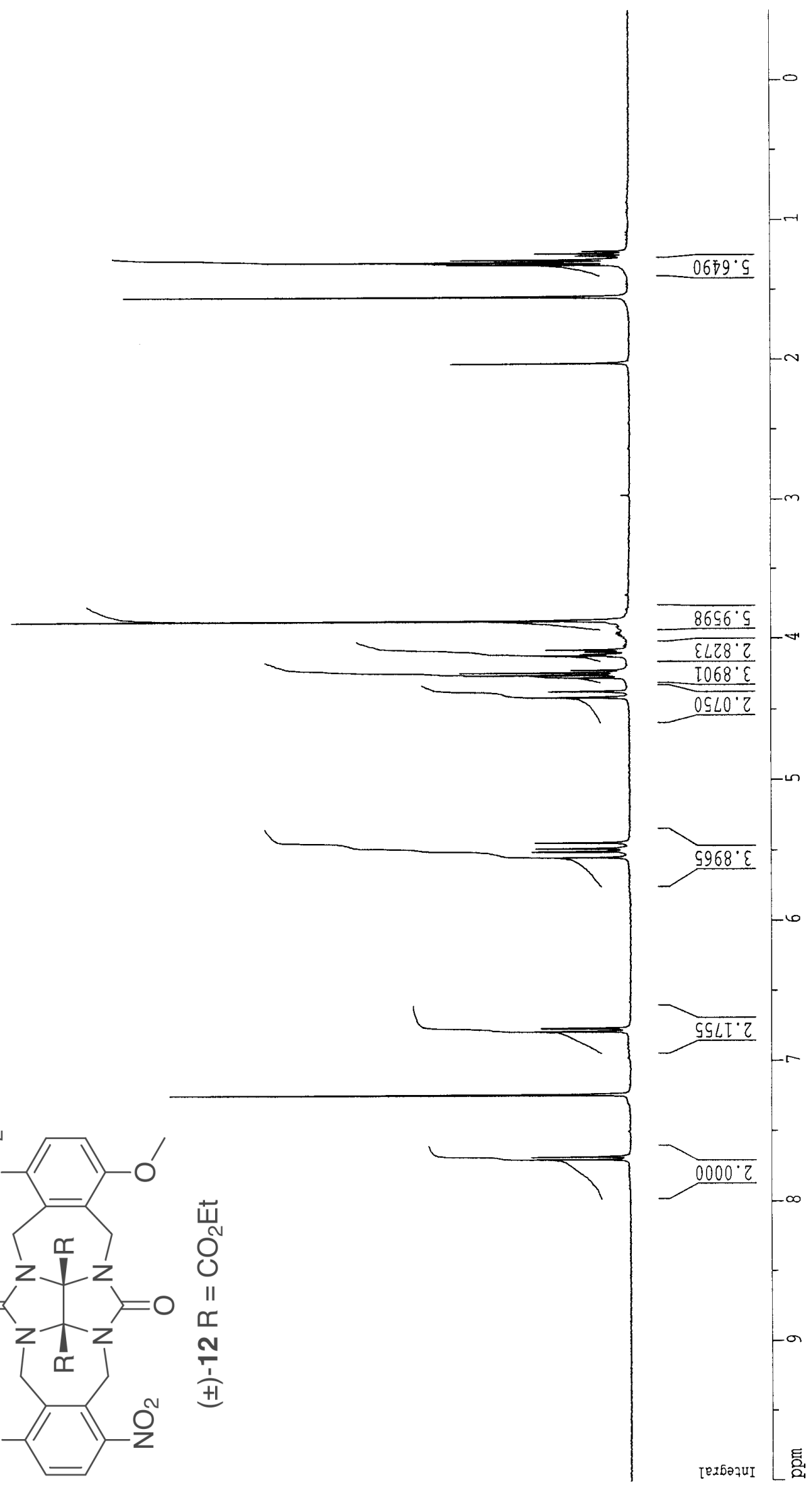




$$
1
$$




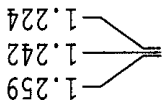

$\angle 0 \varepsilon^{\circ} \varepsilon-$ $\angle E L L^{\circ} \varepsilon>$ 配元 乙ย乙'ฑ $6 \pi 7^{\circ} \pi$ $\angle 9 Z^{\circ} \bar{\square}$ LLC' $T$ LIE' $\overline{-}$

ยโ9'万—

$559^{\circ}$

$980^{\circ} \mathrm{G}$

LZT'S

$922 \cdot c-7$

$\varepsilon 78 \cdot 9 \longrightarrow$

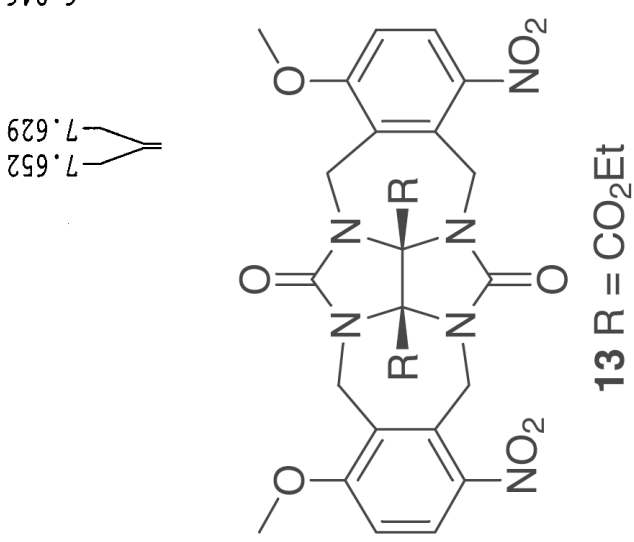

urdd

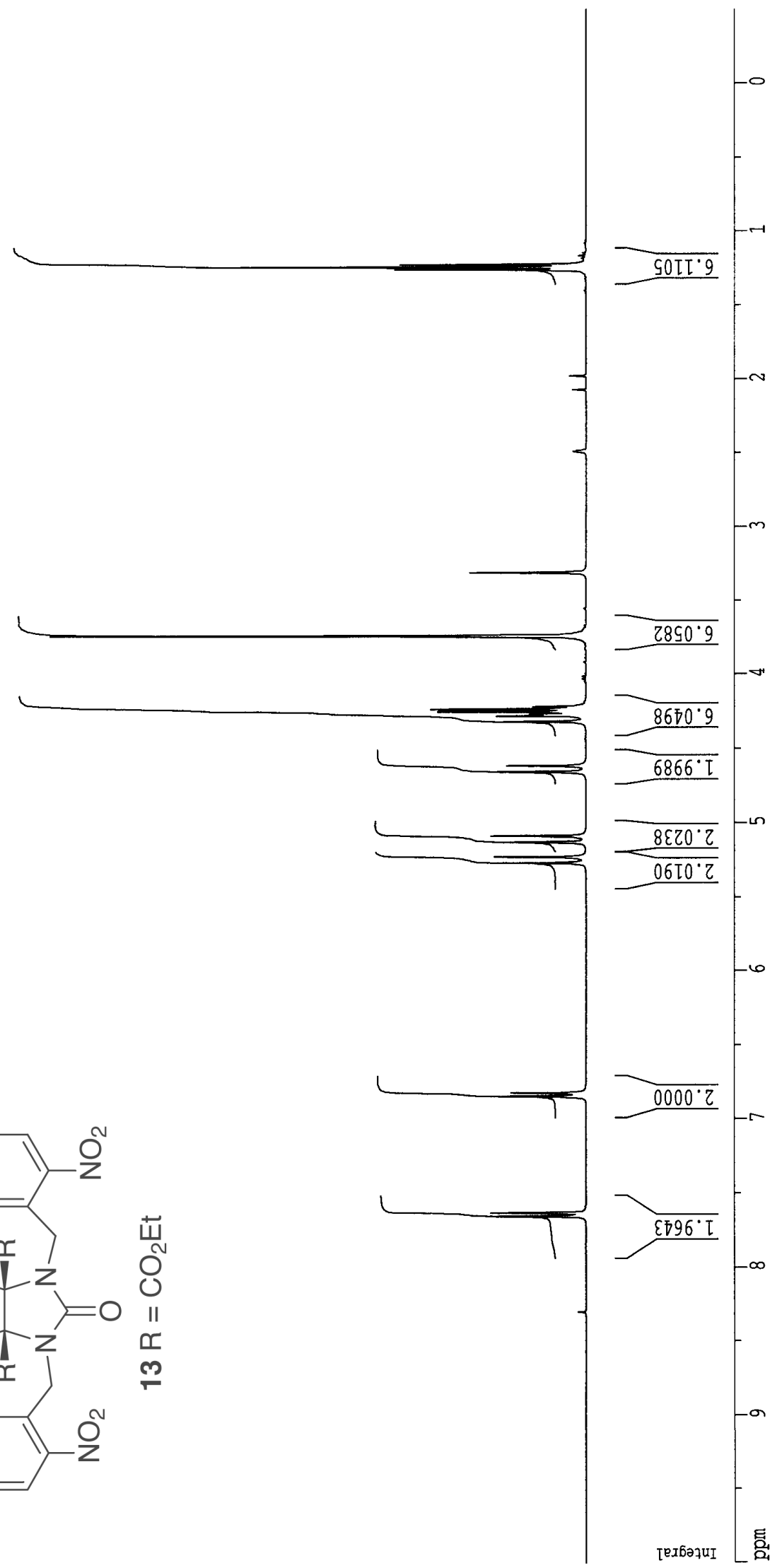



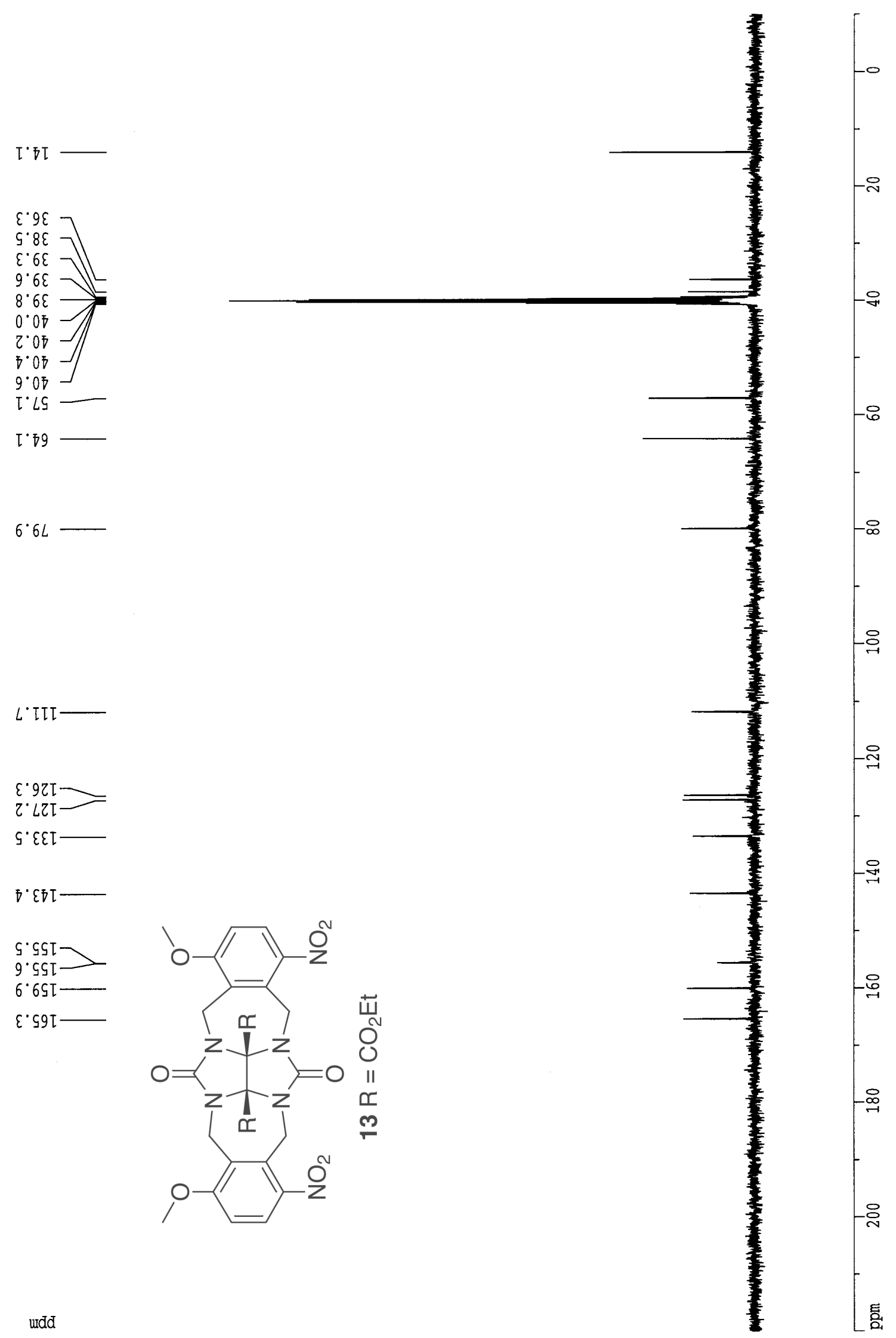

$6.6 L$

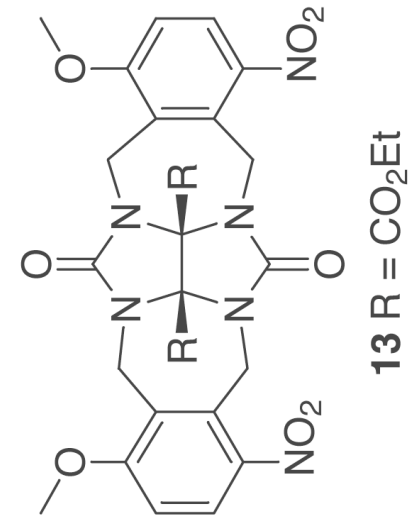

